\title{
Graphene Reinforced Nanocomposites for 3D Printing Applications
}

\author{
María Soria Sánchez, Gerard Tobías Rossell \\ Institute of Material Science of Barcelona, ICMAB (CSIC) \\ Campus de la UAB, 08193, Bellaterra, Barcelona, Spain \\ msoria@icmab.es; gerard.tobias@icmab.es
}

\section{Extended Abstract}

3D printing, also known as Additive Manufacturing (AM) or Rapid Prototyping (RP) is a process for creating 3 dimensional physical objects from a 3D computer digital design. ${ }^{1}$ In recent years, several research groups and companies are working on finding new materials with advanced properties suitable for the development of prototypes. Layered graphene is an emerging material for $3 \mathrm{D}$ printing ${ }^{2}$ due to the combination of its impressive conductivity and the 3D nature of the printed structures, enabling the generation of high surface areas with good electrical properties and hierarchical pore structures, allowing its utilization in the fabrication of electronic devices, like batteries, sensors or RF antennas. The main aim of this work is to study the impact of modified graphene nanoplatelets integrated into the structure of selected polymers matrix. The chemical modification of graphene is expected to improve its integration into commercially available polymers. An improved dispersion has a direct impact on the polymer/graphene interfacial interaction, which has shown to be a critical parameter for the stress transfer from the nanoparticles to the matrix. ${ }^{3,4}$

Polylactic acid (PLA), acrylonitrile butadiene styrene (ABS) and polycaprolactone (PCL) were chosen as matrix since they are one of the most versatile and used polymers in 3D printing. ${ }^{5} \mathrm{UV}$-crosslinkable polydimethylsiloxane (PDMS) was chosen also as a matrix due to its elasticity after curing process, which opens new possibilities in the field of 3D printing since it has not been used for this purpose before. Graphene has been synthesized through reducing lyophilized and non lyophilized both graphene oxide (GO) with hydrazine monohydrate and ascorbic acid (L-AA). Integration of graphenic nanosheets has been carried out by two techniques: intercalation through solution and by melting the polymers in combination with a high shear mixer. Solution method involves evaporation of the solvent and dried, and high shear mixer condition method involves melt mixing. This system has the advantage of not using solvent, which is often considered more economical. Nanocomposites were prepared with graphene loadings of $4 \mathrm{wt} \%$. TGA, Raman, XRD, STEM, SEM, XPS and electrical conductivity measurements are been used for characterizing the materials.

TEM images of the samples synthesized show different size and number of wrinkles on the surface of the graphenic layers due to the chemical treatments applied. Raman and XRD spectroscopies of the graphenic structures confirmed the successfully synthesis of graphene oxide (GO) and reduced graphene oxide (rGO). Raman spectra of the nanocomposites synthesized showed that the integration of rGO into the polymers has been achieved, since the typical bands corresponding to the rGO as well as typical band for each polymer can be observed in the graphs. SEM micrographs and XPS spectroscopy also evidenced a homogeneous dispersion and integration of the graphenic structures into the polymers. Thermal characterization by TGA show an increment in the decomposition temperature of the nanocomposites, which can be translated like an increment in the thermal stability of the nanocomposites, attributed to the effect of the graphenic layers into the polymer which reinforces these polymeric structures.

It has been established that a well dispersed rGO renders not only improves properties in relation to strength and fracture toughness, but also conductivity of the composite, which it's not possible to get by adding GO. Future work will involve the extrusion of the nanocomposites filaments for being used in 3D printing. 


\section{References}

[1] M. Baumers, "Economic Aspects of Additive Manufacturing: Benefits, Costs and Energy Consumption," Ph.D. dissertation, Loughborough University, 2012.

[2] X. Weil et al., "3D Printable Graphene Composite," Sci. Rep., vol. 5, no. 11181, 2015.

[3] L.-C. Tang, et al, "The effect of graphene dispersion on the mechanical properties of graphene/epoxy composites," Carbon, vol. 60, pp. 16-27, 2013.

[4] M. A. Rafiee, et al, "Enhanced Mechanical Properties of Nanocomposites at Low Graphene Content," ACS Nano, vol. 3, no. 12, pp. 3884-3890, 2009.

[5] K. Prashantha et al, "Multifunctional Properties of 3D Printed Poly(lactic acid)/Graphene Ganocomposites by Fused Deposition Modelling," Journal Of Macromolecular Science, Part A: Pure And Applied Chemistry, vol. 54, no. 1, pp. 24-29, 2017. 